\title{
Life Medicaization and Health System, Perspective and Impact
}

\author{
Abdolhassan Kazemi ${ }^{1, *}$ \\ ${ }^{1}$ Infectious and Tropical Diseases Research Center, Tabriz University of Medical Sciences, Tabriz, Iran \\ *Corresponding author: Abdolhassan Kazemi, Infectious and Tropical Diseases Research Center, Tabriz University of Medical Sciences, Tabriz, Iran. E-mail: kazemi1338@Gmail.com
}

Received: 22 Sep 2016

Accepted: 21 Nov 2016

Ppub: 23 Dec 2016

\begin{abstract}
Background: During last several decades a significant number of scientists and sociologists such as Thomas Szasz, Jess R. Pitt, Elliot Fridson, Michael Foucault, Irvin K. Zola, Ivan Illich, and Peter Conrad have warned about medicalization and overmedicalization of health system due to the unacceptable management of this system and non-scientific behavior of medical environment. These theorists and thinkers postulated that medicalization and overmedicalization could be create some forms of socio-medical control of life in which physicians, surgeons and medical authority expanded into domains of everyday existence and life of every persons in human society.

Methods: According the idea of these scientists, human life and conditions come to be identified and explained as medical conditions and then as illness, physical or mental disability, Conditions in need of medical care, etc.

Results: This kind of view to the life and health of human beings and human society has been called "medicalization" and due to medicalization concept, self-identity of people and health decisions could be depend on the prevailing defines of health and illness. Albeit, medicalization and over medicalization in turn also brings over testing, over diagnosis and over treatment to follow. On the other hand, beside of medicalization there are evidences about developing of paramedicalization of life, where personal life of all citizens and also conditions of social health come under the attention of other kind of medicine such as alternative medicine, traditional medicine, herbal medicine, complementary medicine, holistic medicine and so on or even non-medical health approaches.

Conclusions: Therefore, medicalization and paramedicalization sometimes seems contradictory and conflicting, but they could feed each other for disease mongering, market base medicine and albeit they both ensure that questions of life, health, illness, disability,... will stay under control of defining human health conditions according of medicalization, overmedicalization concept.
\end{abstract}

Keywords: Health System; Life Medicalization; Overmedicalization; Overtesting Overdiagnosis; Disease Mongering 